\title{
Plan de marketing 2.0 para impulsar las ventas en franquicia de alimentos
}

\author{
Marketing 2.0 plan to drive food franchise sales
}

Mariana Cabeza

mariana1090@gmail.com

Código ORCID: 0000-0003-3364-9722

Universidad de Carabobo - Venezuela

\section{Dalia Gahona}

daliagahona@gmail.com

Código ORCID: 0000-0001-6052-7275

Universidad de Carabobo - Venezuela

Artículo recibido abril 2019 / Arbitrado en mayo 2019 / Publicado en julio 2019

\section{RESUMEN}

La presente investigación tuvo como objetivo general elaborar un Plan de Marketing 2.0 para una franquicia de comida rápida, ubicada en el Municipio Naguanagua del Estado Carabobo, Venezuela. El estudio presenta la importancia de que una empresa maneje un Plan de Marketing, ya que hoy en día el uso eficiente de las redes sociales es parte de la gestión gerencial para poder impulsar las ventas de la organización. Metodológicamente se apoyó en una investigación descriptiva de campo, enmarcada en un proyecto factible. En virtud de los resultados se realizó la propuesta de un Plan de Marketing 2.0 con la finalidad de mejorar la captación de nuevos clientes y el aumento en el volumen de ventas.

Palabras clave: Marketing; venta; competitividad; globalización y mercado

ABSTRACT

This research had the general objective of preparing a Marketing 2.0 Plan for a fast food franchise, located in the Naguanagua Municipality of Carabobo State, Venezuela. The study shows the importance of a company managing a Marketing Plan, since today the efficient use of social networks is part of managerial management in order to drive sales for the organization. Methodologically it was based on a descriptive field research, framed in a feasible project. By virtue of the results, a proposal for a Marketing 2.0 Plan was made in order to improve the recruitment of new customers and the increase in sales volume.

Key words: Marketing; sales; competitiveness; globalization and market 


\section{INTRODUCCIÓN}

El plan de marketing es la herramienta básica de gestión que debe utilizar toda empresa orientada al mercado que quiera ser competitiva. En su puesta en marcha quedarán fijadas las diferentes actuaciones que deben realizarse en el área del marketing, para alcanzar los objetivos marcados. Este no se puede considerar de forma aislada dentro de la compañía, sino totalmente coordinado y congruente con el plan estratégico, siendo necesario realizar las correspondientes adaptaciones con respecto al plan general de la empresa, ya que es la única manera de dar respuesta válida a las necesidades y temas planteados.

El Marketing 2.0 representa un cambio dramático en beneficio de las búsquedas y compras de bienes y servicios independientemente de la publicidad, campañas de marketing y mensajes. En él, los clientes toman decisiones bajo sus propios términos, apoyándose en las redes de confianza para formar opiniones, como ser amigos o familiares. Se puede decir que ha cambiado los roles del marketing, antes y todavía ahora el marketing lo hacían los directivos y sus agencias, con este significativo cambio el marketing de un futuro muy cercano lo hará cualquiera.

Con la entrada de la nueva era digital y la web 2.0, el marketing digital ha propiciado un nuevo cambio en la concepción y la forma en que los consumidores encuentran, eligen y llevan a cabo la compraventa de bienes y servicios. También ha cambiado la comunicación e interacción de éstos respecto a los negocios a través de internet. La web 2.0 ha permitido que los usuarios se hayan convertido en el epicentro del universo digital.
Éstos pueden comunicarse e interaccionar entre sí gracias a diferentes herramientas (blogs, wikis y redes sociales son las más comunes). Los usuarios han evolucionado y se han transformado en consumidores proactivos (también llamados prosumers) ya que toman las decisiones en base a la información previamente obtenida en internet. Una vez han consumido bienes o servicios, dan su opinión para que el resto de la comunidad la conozca y ésta se beneficie a su vez, en su posible toma de decisiones.

La empresa Papa Jhons ubicada en el Municipio Naguanagua del Estado Carabobo, es una cadena de reparto a domicilio de pizza. La corporación acredita su crecimiento al excelente servicio al cliente, la calidad de sus productos y la simplicidad del menú, en contraste con otras cadenas que se centran en los bajos precios. Es por eso, que se propone desarrollar un plan de marketing 2.0 para implementar las ventas en dicha empresa, aunque muchas empresas suelen pensar que con la creación de un sitio web ya tendrán una gran cantidad de clientes en la puerta de su negocio. Pero en realidad esto no ocurre ya que debe llevarse a cabo una gran investigación y planificación de forma previa. Esta planificación, basada totalmente en el modelo web, debe permitir mejorar la comunicación e interacción con el gran protagonista de la web 2.0, el consumidor.

Dicha planificación, se basa principalmente en el análisis de la situación interno y externo de la empresa. A partir de éste se deben definir una serie de objetivos a cumplir en un determinado tiempo y mediante el uso de los medios digitales. El plan proporciona una dirección marcada al negocio y evita toda improvisación y posibles cambios de rumbo que suelen darse cuando no existe una 
investigación y planificación. A su vez, la correcta implementación de las estrategias digitales implica un ahorro de tiempo y dinero.

Bend Shmit, autor del libro: "El marketing experiencial" nos dice: como conseguir clientes para sentir, pensar, actuar y relacionarse con su empresa y marca" plantea que "el marketing experiencial es un conjunto de políticas y estrategias recientes e innovadoras, centradas en la búsqueda de una nueva fuente de ventaja competitiva basada en la implicación emocional de los clientes y en la creación de experiencias ligadas al producto o servicio" (García, 2010).

Según un proceso de observación, el restaurant Papa Jhons no tiene un plan de marketing definido, no cuenta con estrategias que lo ayuden a difundir y dar a conocer la marca, ni un proceso de comercialización que lo acerque a sus principales consumidores o clientes. Es por eso que surge la inquietud de cómo atraer nuevos clientes, incrementar el consumo de sus productos, aumentar el posicionamiento de la marca y generar la satisfacción de los clientes con el servicio y los productos del restaurante mediante la implementación de estrategias de mercado que involucren experiencias, servicio y calidad, llevando a los investigadores a preguntarse sí la creación de un Plan de Marketing 2.0 aportaría beneficios para lograr crecer en ventas y captar nuevos clientes para la franquicia.

Para la realización de este plan de marketing 2.0 se requiere de información básica tal como, misión y visión de la franquicia, el perfil de sus clientes meta, promociones dentro de las redes, entre otras técnicas que nos permitirán obtener un resultado favorable para los socios ya que contara con más clientes y mayor número de ventas.
Por lo tanto, el objetivo concreto de esta investigación es elaborar un plan de marketing 2.0 para la franquicia Papa Jhons ubicada en el Municipio Naguanagua del Estado Carabobo por lo que es importante obtener la siguiente información:

- ¿A quién va dirigido el producto en internet?

- ¿Cuál será la estrategia de promoción indicada para impulsar la marca?

- ¿Cómo es el alcance del producto al cliente?

- ¿Está registrada la marca en las diferentes redes sociales bajo un mismo nombre para la identificación de la marca?

- ¿Se describen todos los productos al cliente?

Para alcanzar dicho objetivo es importante Investigar las necesidades del mercado real de la franquicia Papa Jhons ubicado en el Municipio Naguanagua del Estado Carabobo, Definir el perfil del cliente en internet de franquicias parecidas a esta, sus debilidades y fortalezas y finalmente Establecer las estrategias del mercado según el plan de marketing 2.0 que favorezcan al mejoramiento de la empresa contribuyendo a la captación de nuevos clientes y al aumento en el volumen de ventas de la empresa.

\section{METODOLOGIA DEL ESTUDIO}

El tipo de investigación que se asume es el denominado estudio descriptivo de campo, El método que se utilizara será cualitativo de naturaleza deductiva ya que pretende conocer cuál es la situación actual de la organización en cuanto a su sistema de marketing para impulsar sus ventas y para ello se utilizará una 
encuesta que tendrá afirmaciones acerca de la percepción de los trabajadores y consumidores en cuanto al proceso de marketing $y$ el investigador será un observador de las respuesta que den los trabajadores y los consumidores de la empresa Papa Jhons.

Sus fuentes son primarias porque se obtiene información directa con los trabajadores y en ocasiones con los consumidores. En lo que a su carácter se refiere es cualitativa y su naturaleza es la encuesta ya que los datos son obtenidos a través de las mismas.

Las unidades de análisis objeto de estudio, será la totalidad de trabajadores de la organización y una porción de consumidores. Todos ellos constituyen la población y la muestra de estudio la cual será tomada en base al universo siempre y cuando sea representativa para la investigación planteada, en ella se generalizarán los resultados. Vale destacar, que la población en la siguiente investigación está conformada por: primero 12 trabajadores, esta población es considerada de tipo finito ya que está constituido por un número que es posible determinarlo. $Y$ en cuanto a los consumidores tomaremos una muestra de 88 consumidores ya que han bajado la cantidad de consumidores que visitan día a día el restaurant.

Es por eso que la muestra es de tipo probabilístico ya que todos los individuos de la población pueden formar parte de la misma. La presente investigación está compuesta por cien (100) sujetos que conforman la muestra total tomada para esta investigación.

\section{RESULTADOS Y DISCUSION}

En la presente investigación se aplica el análisis estadístico de datos, derivado de la introducción de medidas de tendencia central, o promedios, índices de variación o medidas de dispersión, a los datos, para establecer consecuencias que se puedan derivar de estas.

$\mathrm{Se}$ observaron veinticuatro variables referentes a las condiciones actuales de la empresa, agrupadas en seis categorías: características exteriores del local, características interiores del local, características de los alimentos, capacitación del personal de servicio, promoción y recursos informáticos.

Para ello se diseñó una lista de verificación como instrumento y así poder evaluar cada una de las variables utilizando una escala de tres niveles: bueno (3), regular (2) y malo (1). Para otorgar una calificación a los aspectos referentes a las características de los alimentos se observó el comportamiento de los comensales al ver el platillo, buscando percibir signos de aprobación o desagrado.

En cuanto a la evaluación de la capacitación del personal, se observó el comportamiento de los meseros, el cajero y los cocineros. Para el procesamiento de los datos capturados en la lista de verificación se sumaron los valores asignados a cada característica obteniendo así un total por categoría, posteriormente se promediaron para poder comparar los resultados obtenidos. El restaurante Papa Jhons se encuentra ubicado en una zona estratégica, por lo que el número de personas que pasan por la zona es considerable.

El número de mesas es adecuado respecto al tamaño del restaurante y se encuentran distribuidas adecuadamente de forma que permiten el fácil acceso. La plantilla laboral, integrada principalmente por los meseros, quienes tienen el primer contacto con el cliente, están adecuadamente capacitados, son serviciales, cálidos y amistosos. El contacto con el cliente, al momento de ingresar al local es inmediato y una vez ordenado el platillo, en 
promedio demoran entre 15 y 20 minutos para servir el alimento solicitado.

\section{Observación de aspectos internos de la empresa}

El restaurante mantiene finanzas saludables (según lo comentado por el gerente), busca mantener la calidad de sus productos y un precio competitivo que le permita permanecer en la preferencia de los clientes. La empresa cuenta con dos computadoras, una en el área de caja y la otra en la administración, ambas computadoras funcionan adecuadamente $y$ tienen acceso a internet por lo que satisfacen las necesidades actuales de la empresa.

La computadora que se encuentra en caja tiene instalado un punto de venta mediante el cual se registran las transacciones diarias y se elaboran las facturas electrónicas expedidas. La categoría en la que se presenta una debilidad visible es la de promoción debido a que no implementan estrategias constantes para atraer clientes nuevos, la estrategia en la cual se basan es el marketing boca a boca por lo que es una prioridad dar buena atención al cliente.

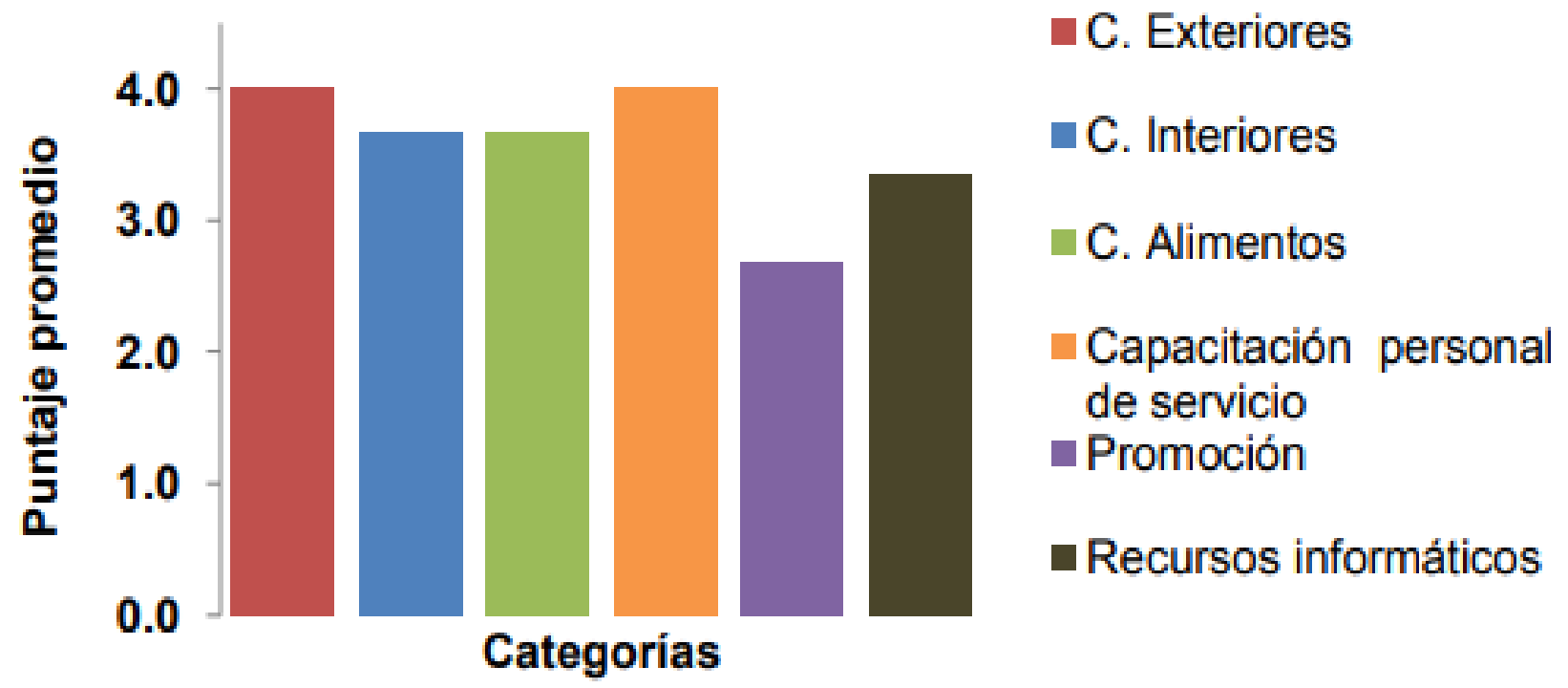

Grafico 1: Comparación de las características de la empresa evaluadas en la observación. Fuente: Cabeza, Gahona (2018)

Se realizó una investigación con el fin de identificar las empresas que se dedican a la preparación de productos como lo que ofrece Papa Jhons en la ciudad y que probablemente representen competencia para el restaurante. En una segunda etapa se realizó una búsqueda de estas en internet, con el fin de conocer su actividad y presencia en este medio. Se utilizó como instrumento una lista de verificación compuesta por once variables agrupadas en dos categorías: la actividad en redes sociales y la actividad en su página web, donde se asignaron los valores de 0 a los restaurantes que no presentan la característica especificada en la lista y 1 a los que sí la presentan. Para la interpretación de los datos, se sumaron los 
valores asignados a cada una de las características por cada empresa. Los resultados obtenidos se compararon para determinar qué restaurante se puede considerar la competencia más fuerte, según su presencia en internet.

La mayoría de las nueve empresas encontradas únicamente aparecen en Facebook y lo utilizan sólo para dar a conocer su menú e instalaciones, pero no generan una interacción con el cliente ni publican temas de interés para el usuario. Sin embargo, ninguno tiene una página de internet que proporcione mayor información sobre el menú que ofrecen o sus promociones, lo que significa que no llegan a clientes que no tienen una cuenta en Facebook.

\section{Propuesta}

A continuación, se anexa la propuesta planteada, que sirve como base para la justificación de dicha propuesta: Desarrollar estrategias de Marketing 2.0 como herramienta para mejorar la participación en el mercado, posicionamiento e incremento de las ventas de la empresa Papa Jhons por medio de una página web y redes sociales.
Basado en el análisis de los resultados del diagnóstico interno $(\mathrm{PCl})$ y de la entrevista realizada a los clientes de la Empresa Papa Jhons, donde se apreció diversas fallas que tiene la organización para adaptarse a las tendencias tecnológicas; donde se determinó los gustos y preferencias que desean encontrar de un proveedor en la web; se procedió a diseñar estrategias de Marketing 2.0 como herramienta para expandir la cobertura en el mercado la empresa bajo estudio.

Esta propuesta está dirigida a mejorar la participación, posicionamiento e incremento de las ventas de la empresa, mediante la promoción de los productos y servicios con opción de compras en Internet. Dicha propuesta busca mantener a los clientes actuales satisfechos, dándoles una nueva opción para adquirir los productos y enterarse de las novedades de Papa Jhons; a su vez ayudaría a captar clientes potenciales, ya que pueden encontrar a la empresa por los diferentes buscadores de Internet, generando altos resultados comerciales. 
Mariana Cabeza y Dalia Gahona

Cuadro 1. Propuesta

\begin{tabular}{|c|c|c|c|}
\hline Estrategia & Táctica & Acción & Responsable \\
\hline $\begin{array}{l}\text { Desarrollo estrategias de } \\
\text { Marketing } 2.0 \text { como } \\
\text { herramienta para mejorar la } \\
\text { participación en el mercado e } \\
\text { incremento de las ventas de la } \\
\text { empresa Papa Jhons }\end{array}$ & $\begin{array}{l}\text { Contratar a un } \\
\text { diseñador Web para } \\
\text { crear un sitio en } \\
\text { Internet }\end{array}$ & $\begin{array}{l}\text { Utilizar esta } \\
\text { herramienta como } \\
\text { medio para } \\
\text { promocionar y } \\
\text { comercializar } \\
\text { productos y } \\
\text { servicios }\end{array}$ & $\begin{array}{l}\text { Diseñador Web } \\
\text { y Gerente de } \\
\text { Ventas }\end{array}$ \\
\hline $\begin{array}{l}\text { Posicionamiento en los } \\
\text { principales buscadores de } \\
\text { Internet }\end{array}$ & $\begin{array}{l}\text { Contratar a } \\
\text { un diseñador } \\
\text { Web para } \\
\text { posicionar la } \\
\text { empresa en } \\
\text { Internet }\end{array}$ & $\begin{array}{l}\text { Ubicar a la } \\
\text { empresa entre las } \\
\text { principales } \\
\text { opciones de } \\
\text { búsqueda }\end{array}$ & Diseñador Web \\
\hline $\begin{array}{l}\text { Creación una cuenta en las } \\
\text { redes sociales }\end{array}$ & $\begin{array}{l}\text { Contratar a un } \\
\text { diseñador Web para } \\
\text { diseñar y abrir un } \\
\text { cuenta en Twitter y } \\
\text { Facebook }\end{array}$ & $\begin{array}{l}\text { Promocionar los } \\
\text { productos y } \\
\text { servicios que } \\
\text { ofrece la empresa }\end{array}$ & $\begin{array}{l}\text { Diseñador Web } \\
\text { y Gerente de } \\
\text { Ventas }\end{array}$ \\
\hline $\begin{array}{l}\text { Incentivo a los clientes de Papa } \\
\text { Jhons a realizar pedidos y/o } \\
\text { compras en la web }\end{array}$ & $\begin{array}{l}\text { Enviar } \\
\text { información } \\
\text { a los clientes } \\
\text { reales sobre } \\
\text { la existencia } \\
\text { de la página } \\
\text { web }\end{array}$ & $\begin{array}{c}\text { Redactar } \\
\text { información } \\
\text { llamativa para } \\
\text { captar la atención } \\
\text { del cliente. }\end{array}$ & Gerente de Ventas \\
\hline $\begin{array}{l}\text { Facilidad de realizarlos } \\
\text { pedidos y/o compra a los } \\
\text { clientes reales y potenciales, } \\
\text { proporcionándoles el } \\
\text { portafolio de productos en la } \\
\text { web y medios de pago en la } \\
\text { web }\end{array}$ & $\begin{array}{l}\text { Enviar por correo } \\
\text { electrónico las } \\
\text { promociones } \\
\text { de productos } \\
\text { y servicios }\end{array}$ & $\begin{array}{l}\text { Enviar por correo } \\
\text { electrónico, } \\
\text { banner llamativos } \\
\text { donde explique } \\
\text { los pasos a seguir } \\
\text { para realizar } \\
\text { pedidos y/o } \\
\text { compras }\end{array}$ & $\begin{array}{l}\text { Diseñador Web } \\
\text { y Gerente de } \\
\text { Ventas }\end{array}$ \\
\hline $\begin{array}{l}\text { Ofrecimiento de seguridad a } \\
\text { los clientes, mediante un } \\
\text { modelo de compra } \\
\text { confidencial al momento de } \\
\text { adquirir los productos con } \\
\text { diferentes modalidades de } \\
\text { pago }\end{array}$ & $\begin{array}{l}\text { Ofrecer un } \\
\text { espacio } \\
\text { confidencial } \\
\text { vía web a los } \\
\text { clientes } \\
\text { reales de la } \\
\text { empresa }\end{array}$ & $\begin{array}{c}\text { Crear } \\
\text { comunicado a través } \\
\text { de correo } \\
\text { electrofónico } \\
\text { para certificar } \\
\text { que pueden } \\
\text { realizar pedidos }\end{array}$ & $\begin{array}{l}\text { Diseñador Web } \\
\text { y Gerente de } \\
\text { Ventas }\end{array}$ \\
\hline
\end{tabular}

Fuente: Cabeza, Gahona (2018) 


\section{CONCLUSIONES}

Como se ha podido comprobar, un plan de marketing ayuda a que una empresa mejore su situación en su correspondiente entorno digital. Genera una dirección clara hacía donde deben ir los objetivos y la forma de conseguirlos mediante el uso de técnicas y herramientas. La planificación aporta creatividad generada a partir del análisis de los competidores y los procesos de mejora de contenidos planteados a partir de éste. Además, el plan permite el desarrollo y la consolidación de la marca.

El desarrollo web y el posicionamiento web son disciplinas de cambio y mejora constante. En muchos casos se plantean estrategias 0 tácticas que una vez implementadas, éstas pueden no ser las más adecuadas, con el tiempo quedan obsoletas, se realizan con nuevas herramientas 0 simplemente se pueden solucionar con otros métodos más sencillos a los escogidos.

La formación impartida ha permitido que el cliente comprenda el porqué de la realización de determinadas acciones en el sitio web. Desde un principio, al optimizar el sitio web el cliente daba la negativa respecto a la incorporación de determinadas palabras clave. Una vez explicados los motivos, el cliente ha aceptado determinados cambios, aunque para otros ha priorizado en la estética antes que en el posicionamiento. Se ha generado una cohesión estratégica para poder resolver los objetivos planteados. Gracias a la comunicación con el cliente se han planteado vías de contacto que han permitido trabajar en los contenidos.

En todo momento ha habido un contacto $y$ un desarrollo de trabajo mutuo para alcanzar los objetivos propuestos y esto es lo que el cliente debe hacer a su vez con su personal. Se ha incidido en la importancia de que el cliente debe comenzar a planificar el tiempo de trabajo y destinar parte de éste a mejorar el marketing digital con el fin de escalar posiciones dentro de su entorno digital. Si la situación actual mejora, el cliente debe optar por nuevas vías de comunicación que le aporten difusión tales como la creación de un blog o la incorporación en nuevas redes sociales como twitter o Instagram. Por lo tanto, se ha enseñado al cliente métodos y técnicas para mejorar su situación actual, pero también se le ha dado la fórmula para continuar mejorando su posición en futuro.

\section{REFERENCIAS}

Bespokely Digital. 5 pasos para crear un plan de marketing digital [en línea].

Balestrini (2001). Cómo se elabora un proyecto de investigación. Editorial Person Prentice Hill. México

Bueno, Carolina (2011). Normativa para los Trabajos de Investigación de la Facultad de Ciencias Económicas $Y$ sociales de la Universidad de Carabobo. Departamento de Publicaciones Facultad de Ciencias Económicas y Sociales

Elosegui, Tristán. "Cómo definir los objetivos de una estrategia online". Tristán Elosegui: marketing online, analítica web y social media

Espinoza, Roberto (2013). La Matriz De Análisis DAFO (FODA). Presentación en Línea. Disponible: http://robertoespinosa.es/2013/07/29/lamatriz-de-analisis-dafo-foda/. Consulta: 2018, octubre 21

Ezquerro, Daniel." Cómo analizar a tu competencia en 5 pasos". 40 de fiebre

Fuentes (2005), Plan estratégico para aumentar las ventas de la empresa Servipaco, C.A. 
tomando en cuenta el mercado de clientes reales y potenciales

Gómez, Marcelo (2006). Introducción a la Metodología Científica. Libro en Línea.Disponible:https://books.google.co.ve /books?id=9UDXPe4U7aMC\&pg=PA121\&d $q=$ Recoleccion + de + Datos\&hl=es419\&sa $=$ $\mathrm{X} \&$ redir_esc $=\mathrm{y} \# \mathrm{v}=$ onepage $\& \mathrm{q}=$ Recoleccio n\%20de\%20Datos\&f=false. Consulta: 2018, diciembre 18

Fachenda (2008), El Comercio Electrónico no es solo Internet. Universidad Simón Bolívar

Hernández Díaz, Alfredo. "Plan y marketing digital: la pareja perfecta 2.0". Alfredo Hernández Díaz: Marketing digital

Hurtado de Barrera, Jacqueline (2008). La Población y Muestra. Artículo en Línea. Disponible:

http://msctecnologiaeducativa3.blogspot.c om/p/poblacion-y-muestra_19.html.

Consulta: 2018, noviembre 20

Jodar, Juan. "Cómo hacer el mejor análisis de tu competencia online". Saltando trenes: blog de marketing online y publicidad online

Kotler (2002). Dirección de Marketing. Pearson Prentice Hall. $8^{\mathrm{a}}$ Edición

Kotler y Arm Strong (2008). Principios del Marketing. Editorial Pearson Prentice Hall. $12^{\mathrm{a}}$ Edición. USA
Ronda, Guillermo (2002). Un concepto de estrategia. Presentación en Línea. Disponible:

https://www.gestiopolis.com/un-conceptode-estrategia/. Consulta: 2018, febrero 25

Maldon Agencia. "Cómo crear tu primer plan de marketing digital". Maldon

Núñez, Vilma. Cómo crear un plan de marketing online [en línea]. Vilma Núñez. Pendino, Sebastián "Cómo hacer un análisis de la competencia paso a paso". Sebastián Pendino

Sánchez, Joana. "10 pasos para crear una estrategia digital de éxito". Líderes digitales

Sancho, Loles. "5 tipos de estrategia de marketing online". Oreste social media. Consulta: 18 febrero 2018

Stanton, Etzel y Walter (2000). Fundamentos del Marketing. $11^{\text {a }}$ Edición, Editorial Mc Graw Ha ll. México

Santiago, Ignacio. "Cómo medir y mejorar la velocidad de carga de tu página web". Ignacio Santiago

Rodríguez (2011). Plan estratégico para aumentar las ventas de la empresa Fuller Interamericana C.A. tomando en cuenta el mercado de clientes reales y potenciales 